\title{
In vivo Imaging of Kidney using Fluorescent Markers with 2 Photon Microscopy
}

\author{
Robert L. Bacallao,* and Ruben M Sandoval* \\ Department of Medicine, Division of Nephrology, Indiana University School of \\ Medicine, Indianapolis, IN 46202
}

Renal function is a combination of glomerular filtration of the blood and tubular modification of the filtrate. It is hard to appreciate this dynamic process from the typical 2 dimensional images of fixed tissue. There are three components to this process, a) renal blood flow, b) glomerular filtration and c) tubular re-absorption and secretion. These normal features can now be directly evaluated as well as pathological processes in rodent kidney such as: altered filtration properties, glomerulosclerosis, tubule endocytosis, exocytosis, intracellular trafficking, apoptosis, increased endothelial permeability, vasoconstriction, epithelial membrane blebbing and mitochondrial function. These processes are viewed in near real time since there are a number of excellent fluorescent markers that can be used in vivo, in combination with 2 photon microscopy (1). The anesthetized rat can be placed directly on the microscope with the kidney exteriorized (Figure 1). Renal tubules are responsible for metabolism and clearing of many different drugs, some of which are toxic to the kidney. For example, Gentamicin, a nephrotoxic antibiotic can be directly visualized as it is filtered, passing through the proximal then distal tubules and finally through the collecting ducts (Figure 2) however some of the gentamycin is endocytosed by the proximal tubule, where it reaches toxic levels (2). Using fluorescent dyes also allows an evaluation of blood flow including the determination of flow rate by evaluating the transit time for RBCs to pass through capillaries and for the determination of plasma clearance rate (Figure 3) by following the disappearance of FITC inulin from the blood (3). Details of in vivo renal imaging methodology have been clearly described so that any lab with 2 photon microscopy capability can perform these types of studies (4).

\section{References}

1. Dunn KW, Sandoval RM, Kelly KJ, Dagher PC, Tanner GA, Atkinson SJ, Bacallao RL, Molitoris BA. Functional studies of the kidney of living animals using multicolor two-photon microscopy. Am J Physiol Cell Physiol. 2002;283:C905-916.

2. Sandoval RM, Molitoris BA. Gentamicin traffics retrograde through the secretory pathway and is released in the cytosol via the endoplasmic reticulum. Am J Physiol Renal Physiol. 2004;286:F617-624

3. Yu W, Sandoval RM, Molitoris BA. Rapid determination of renal filtration function using an optical ratiometric imaging approach. Am J Physiol Renal Physiol. 2007 ;292:F1873-1880.

4. Sandoval RM, Molitoris BA. Quantifying endocytosis in vivo using intravital twophoton microscopy. Methods Mol Biol. 2008;440:389-402. 


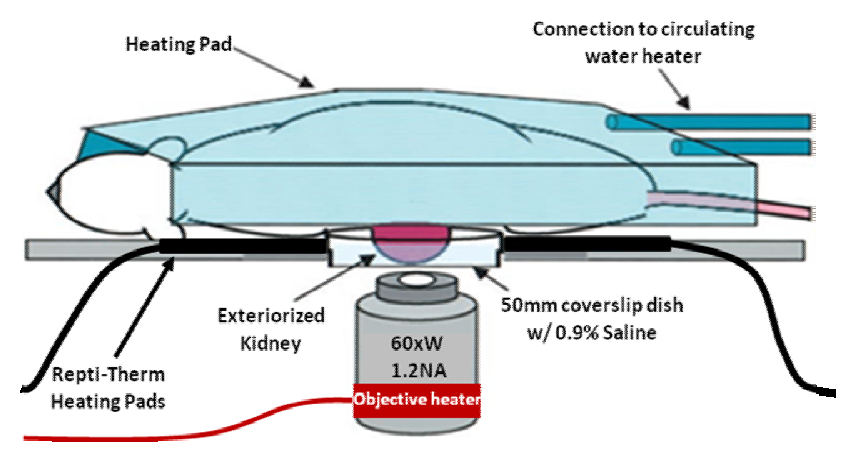

Figure 1. After the rat is anesthetized, a catheter is placed in the femoral vein, the kidney is exposed through a surgical incision and carefully placed in a coverslip bottom dish in $1 \mathrm{XNaCl}$. The rat is kept warm with a heating pad. (modified from Reference 1)
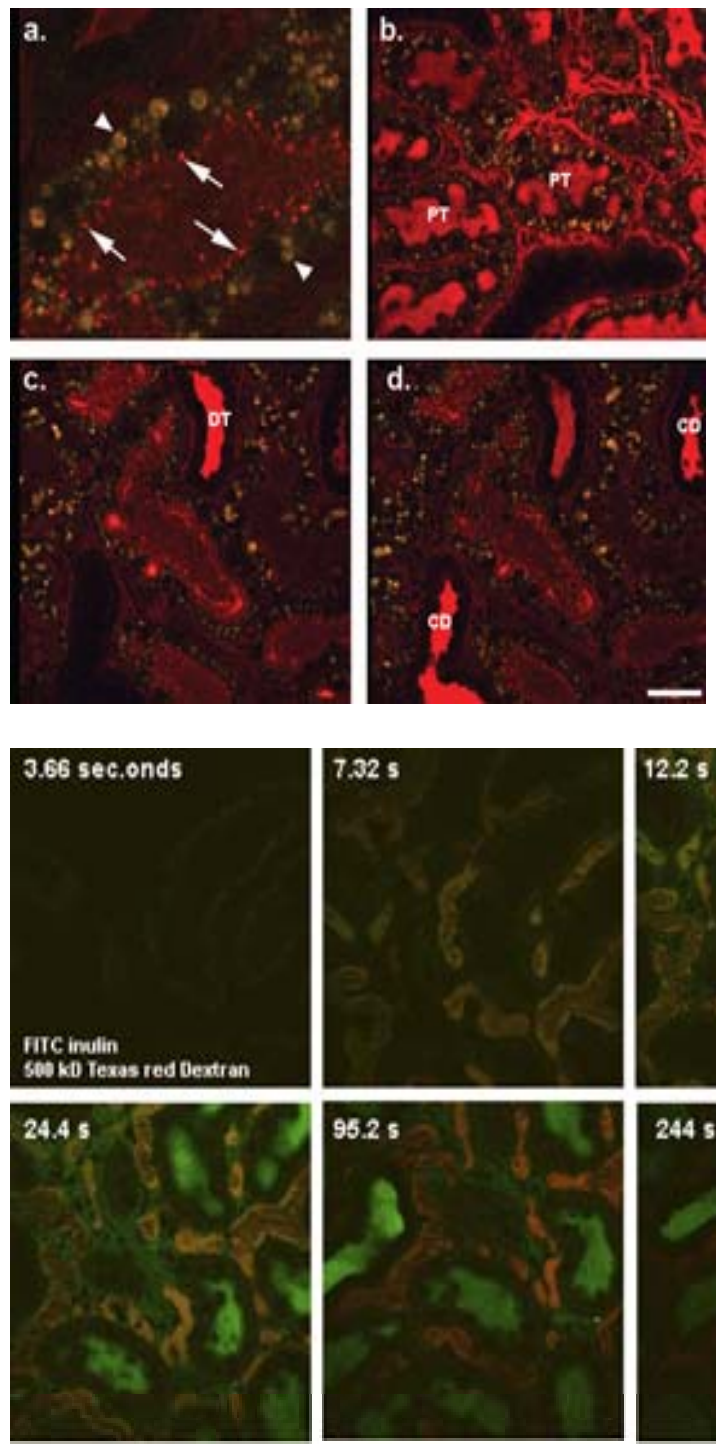

Figure 2. Using a timed series of images (b.-d.) from an intact rat given Texas-red gentamicin i.v., the gentamicin was found in proximal tubules (PT in b.) at $1 \mathrm{~min}$. and then seen in distal tubules (DT in c.) at 3 minutes and shortly thereafter in the collecting ducts (CD in d.). However, some Texas-red gentamicin was seen in the endosomes of the proximal tubule (arrows in a.). The retention of gentamicin in PTs intitiates in nephrotoxic affect on this segment. Scale bar $=40 \mathrm{um}$ (modified from reference 1.)
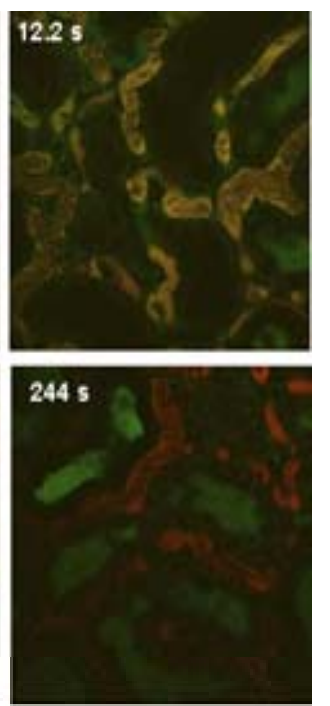

Figure 3. A timed series of images from an intact rat given FITCinulin (filtered) and Texas-red $500 \mathrm{kD}$ dextran (not filtered). The green and red are initially together in blood vessels, but as the inulin is filtered (and is seen in the renal tubules, green) it disappears from the blood leaving the Texas-red in the vessels. The disappearance of the FITC from the blood can be used to determine the clearance rate (GFR). (modified from reference 3 ) 